\title{
DEVELOPMENT
}

\section{Adult Leydig cell maintenance}

New data from an in vitro culture model of rat seminiferous tubules elucidate how the development of new adult Leydig cells from stem Leydig cells (SLCs) is regulated. The study found that desert hedgehog protein is central to the proliferation and differentiation of the stem cells, committing these progenitors to the Leydig cell lineage. In addition, Thy- 1 antigen (CD90) emerged as a specific surface marker of the SLCs, enabling selective cell isolation.

Leydig cells in the testes are the principal source of testosterone. Apart from its elementary function in the development of the male reproductive system, lack of testosterone in adult men has been connected to losses in bone density and muscle mass, as well as decreased immune and cognitive functions. Hence, understanding Leydig cell development might enable novel therapeutic approaches in the future. The adult Leydig cell population is believed to be maintained by stem cells, rather than originating from fetal progenitors. Xiaoheng Li and colleagues used their in vitro rat seminiferous tubule culture model to investigate factors involved in Leydig cell expansion. In this system, stem cells predominantly proliferate in the first week of culture, followed by differentiation in the subsequent weeks, similar to events in vivo.

The researchers incubated tubules with different factors that had previously been implicated in Leydig cell development in the presence of luteinizing hormone. Incubation with the desert hedgehog protein agonist $\mathrm{SAG}$ resulted in the highest levels of testosterone produced after 3 weeks, regardless whether cells had been incubated during week 1 only or during weeks 2 and 3 only.

Luteinizing hormone is a key factor in Leydig cell development, but SLCs do not express the luteinizing hormone receptor; hence, the researchers tested whether the presence of SAG in weeks 2 and 3 would result in increased testosterone production in the absence of luteinizing hormone: at the end of week 3 , after brief $(\sim 2 \mathrm{~h})$ addition of luteinizing hormone, testosterone production of tubules incubated with SAG was significantly higher than in controls. Further experiments in the absence of Sertoli cells, which might be involved in Leydig cell development via paracrine factors, showed that incubation with SAG in the presence of luteinizing hormone resulted in testosterone production, pointing towards a central role of desert hedgehog protein in SLC proliferation and differentiation. To corroborate this finding, the team identified CD90 as a unique marker for the SLC population and showed that only $\mathrm{CD} 90^{+}$cells isolated from disaggregated tubules ( $~ 0.4 \%$ of all cells) produced testosterone at week 3 after incubation with SAG in the presence of luteinizing hormone.

Clemens Thoma

ORIGINAL ARTICLE Li, X. et al. Regulation of seminiferous tubule-associated stem Leydig cells in adult rat testes. Proc. Natl Acad. Sci. USA http:// dx.doi.org/10.1073/pnas.1519395113 (2016) 